\title{
Correction to: Multivariate approximations in Wasserstein distance by Stein's method and Bismut's formula
}

\author{
Xiao Fang ${ }^{1} \cdot$ Qi-Man Shao ${ }^{1} \cdot$ Lihu $\mathrm{Xu}^{2,3}$
}

Published online: 11 July 2019

๑) Springer-Verlag GmbH Germany, part of Springer Nature 2019

\section{Correction to: Probability Theory and Related Fields https://doi.org/10.1007/s00440-018-0874-5}

We write this note to correct $[1,(\mathbf{6 . 9}),(\mathbf{6 . 1 3}),(\mathbf{7 . 1}),(\mathbf{7 . 2})]$ because there was one term missed in [1, (6.9)]. To estimate this missed term, we need to add an extra condition to [1, Assumption 2.1]:

Assumption $2.1 \mathrm{~g} \in \mathcal{C}^{3}\left(\mathbb{R}^{d}, \mathbb{R}^{d}\right)$, and there exist $\theta_{0}>0$ and $\theta_{1}, \theta_{2}, \theta_{3}, \theta_{3}^{\prime} \geq 0$ such that the conditions (2.3) and (2.4) in [1] hold, i.e.,

$$
\begin{array}{cl}
\left\langle u, \nabla_{u} g(x)\right\rangle \leq-\theta_{0}\left(1+\theta_{1}|x|^{\theta_{2}}\right)|u|^{2}, & \forall u, x \in \mathbb{R}^{d} ; \\
\left|\nabla_{u_{1}} \nabla_{u_{2}} g(x)\right| \leq \theta_{3}\left(1+\theta_{1}|x|\right)^{\theta_{2}-1}\left|u_{1}\right|\left|u_{2}\right|, & \forall u_{1}, u_{2}, x \in \mathbb{R}^{d} .
\end{array}
$$

and additionally,

$$
\left|\nabla_{u_{1}} \nabla_{u_{2}} \nabla_{u_{3}} g(x)\right| \leq \theta_{3}^{\prime}(1+|x|)^{\theta_{2}-2}\left|u_{1}\right|\left|u_{2}\right|\left|u_{3}\right|, \quad \forall u_{1}, u_{2}, u_{3}, x \in \mathbb{R}^{d}
$$

Under the above-strengthened Assumption 2.1, all the conclusions and examples in [1] still hold true, except that all the constants $C_{\theta}$ therein will depend on the constants in the new assumption.

The original article can be found online at https://doi.org/10.1007/s00440-018-0874-5.

\section{Lihu Xu}

lihuxu@umac.mo

Xiao Fang

xfang@sta.cuhk.edu.hk

Qi-Man Shao

qmshao@sta.cuhk.edu.hk

1 Department of Statistics, The Chinese University of Hong Kong, Shatin, N.T., Hong Kong

2 Department of Mathematics, Faculty of Science and Technology, University of Macau, Av. Padre Tomás Pereira, Taipa, Macau, China

3 Zhuhai UM Science and Technology Research Institute, Zhuhai, China 
Before correcting [1, (6.9), (6.13), (7.1), (7.2)], let us recall some notations in [1], give the missed term, and prove an auxiliary lemma. Let $u, u_{1}, u_{2} \in \mathbb{R}^{d}$, recall

$$
\mathcal{I}_{u}^{x}(t)=\frac{1}{\sqrt{2} t} \int_{0}^{t}\left\langle\nabla_{u} X_{s}^{x}, \mathrm{~d} B_{s}\right\rangle, \quad \mathcal{I}_{u_{1}, u_{2}}^{x}(t)=\mathcal{I}_{u_{1}}^{x}(t) \mathcal{I}_{u_{2}}^{x}(t)-D_{V_{2}} \mathcal{I}_{u_{1}}^{x}(t)
$$

with $V_{i, t}=\int_{0}^{t} v_{i}(s) \mathrm{d} s$ and $v_{i}(s)=\frac{1}{\sqrt{2} t} \nabla_{u_{i}} X_{s}^{x}$ for $0 \leq s \leq t$ and $i=1,2$, see [1, (5.12),(5.13)]. The missed term is defined by

$$
\mathcal{R}_{u_{1}, u_{2}}^{x}(t):=\nabla_{u_{2}} \nabla_{u_{1}} X_{t}^{x}-D_{V_{2}}\left(\nabla_{u_{1}} X_{t}^{x}\right)
$$

Lemma 0.1 We have

$$
\begin{aligned}
\left|\mathcal{R}_{u_{1}, u_{2}}^{x}(t)\right| & \leq C_{\theta}\left|u_{2}\right|\left|u_{1}\right|, \\
\left|\nabla_{u_{3}} \mathcal{R}_{u_{1}, u_{2}}^{x}(t)\right| & \leq C_{\theta}\left|u_{3}\right|\left|u_{2}\right|\left|u_{1}\right|, \\
\left|D_{V_{3}} \mathcal{R}_{u_{2}, u_{1}}^{x}(t)\right| & \leq C_{\theta}\left|u_{3}\right|\left|u_{2}\right|\left|u_{1}\right|,
\end{aligned}
$$

for all $u_{1}, u_{2}, u_{3}, x \in \mathbb{R}^{d}$.

Proof The first bound follows immediately from [1, (5.7),(5.17)]. It is easy to check that $\nabla_{u_{3}} \nabla_{u_{2}} \nabla_{u_{1}} X_{t}$ satisfies the equation

$$
\frac{\mathrm{d}}{\mathrm{d} t} \nabla_{u_{3}} \nabla_{u_{2}} \nabla_{u_{1}} X_{t}=\nabla g\left(X_{t}\right) \nabla_{u_{3}} \nabla_{u_{2}} \nabla_{u_{1}} X_{t}+\nabla^{2} g\left(X_{t}\right) \mathcal{R}_{1}(t)+\nabla^{3} g\left(X_{t}\right) \mathcal{R}_{2}(t),
$$

where $\mathcal{R}_{1}$ and $\mathcal{R}_{2}$ are terms about first and second-order derivatives of $X_{t}$. By [1, (5.6), (5.7)], we have

$$
\left|\mathcal{R}_{1}(t)\right| \leq C_{\theta}\left|u_{1}\right|\left|u_{2}\right|\left|u_{3}\right|, \quad\left|\mathcal{R}_{2}(t)\right| \leq C_{\theta}\left|u_{1}\right|\left|u_{2}\right|\left|u_{3}\right|
$$

Differentiating $\left|\nabla_{u_{3}} \nabla_{u_{2}} \nabla_{u_{1}} X_{t}\right|^{2}$ with respect to $t$ and using the above two bounds, we can prove by the same argument as showing [1, (5.7)]

$$
\left|\nabla_{u_{3}} \nabla_{u_{2}} \nabla_{u_{1}} X_{t}\right| \leq C_{\theta}\left|u_{1}\right|\left|u_{2}\right|\left|u_{3}\right|
$$

Similarly, first finding the differential equations of $\nabla_{u_{3}} D_{V_{2}} \nabla_{u_{1}} X_{t}, D_{V_{3}} \nabla_{u_{2}} \nabla_{u_{1}} X_{t}$, $D_{V_{3}} D_{V_{2}} \nabla_{u_{1}} X_{t}$, and then using the same argument as above, we get

$$
\begin{aligned}
& \left|\nabla_{u_{3}} D_{V_{2}} \nabla_{u_{1}} X_{t}\right| \leq C_{\theta}\left|u_{1}\right|\left|u_{2}\right|\left|u_{3}\right|, \\
& \left|D_{V_{3}} \nabla_{u_{2}} \nabla_{u_{1}} X_{t}\right| \leq C_{\theta}\left|u_{1}\right|\left|u_{2}\right|\left|u_{3}\right|, \\
& \left|D_{V_{3}} D_{V_{2}} \nabla_{u_{1}} X_{t}\right| \leq C_{\theta}\left|u_{1}\right|\left|u_{2}\right|\left|u_{3}\right| .
\end{aligned}
$$

Collecting the previous estimates, we immediately obtain the other two estimates in the lemma. 
Correction to $[1,(6.9),(6.13)]$ : The original $[1,(6.9),(6.13)]$ should be corrected as

$$
\nabla_{u_{2}} \nabla_{u_{1}} \mathbb{E}\left[\phi\left(X_{t}^{x}\right)\right]=\mathbb{E}\left[\nabla_{u_{1}} \phi\left(X_{t}^{x}\right) \mathcal{I}_{u_{2}}^{x}(t)\right]+\mathbb{E}\left[\nabla \phi\left(X_{t}^{x}\right) \mathcal{R}_{u_{1}, u_{2}}^{x}(t)\right]
$$

and

$$
\begin{aligned}
\nabla_{u_{2}} \nabla_{u_{1}} f(x)= & \int_{0}^{\infty} e^{-t} \mathbb{E}\left\{\left[\nabla_{u_{1}} f\left(X_{t}^{x}\right)-\nabla_{u_{1}} h\left(X_{t}^{x}\right)\right] \mathcal{I}_{u_{2}}^{x}(t)\right\} \mathrm{d} t \\
& +\int_{0}^{\infty} e^{-t} \mathbb{E}\left\{\left[\nabla f\left(X_{t}^{x}\right)-\nabla h\left(X_{t}^{x}\right)\right] \mathcal{R}_{u_{2}, u_{1}}^{x}(t)\right\} \mathrm{d} t
\end{aligned}
$$

for $u_{1}, u_{2}, x \in \mathbb{R}^{d}$.

Proof We have

$$
\nabla_{u_{2}} \nabla_{u_{1}} \mathbb{E}\left[\phi\left(X_{t}^{x}\right)\right]=\mathbb{E}\left[\nabla^{2} \phi\left(X_{t}^{x}\right) \nabla_{u_{2}} X_{t}^{x} \nabla_{u_{1}} X_{t}^{x}\right]+\mathbb{E}\left[\nabla \phi\left(X_{t}^{x}\right) \nabla_{u_{2}} \nabla_{u_{1}} X_{t}^{x}\right],
$$

By [1, (5.14), (5.9), (5.11)],

$$
\begin{aligned}
\mathbb{E}\left[\nabla^{2} \phi\left(X_{t}^{x}\right) \nabla_{u_{2}} X_{t}^{x} \nabla_{u_{1}} X_{t}^{x}\right] & =\mathbb{E}\left[\nabla^{2} \phi\left(X_{t}^{x}\right) D_{V_{2}} X_{t}^{x} \nabla_{u_{1}} X_{t}^{x}\right] \\
& =\mathbb{E}\left[D_{V_{2}}\left(\nabla \phi\left(X_{t}^{x}\right)\right) \nabla_{u_{1}} X_{t}^{x}\right] \\
& =\mathbb{E}\left[D_{V_{2}}\left(\nabla \phi\left(X_{t}^{x}\right) \nabla_{u_{1}} X_{t}^{x}\right)\right]-\mathbb{E}\left[\nabla \phi\left(X_{t}^{x}\right) D_{V_{2}}\left(\nabla_{u_{1}} X_{t}^{x}\right)\right] \\
& =\mathbb{E}\left[\nabla_{u_{1}} \phi\left(X_{t}^{x}\right) \mathcal{I}_{u_{2}}^{x}(t)\right]-\mathbb{E}\left[\nabla \phi\left(X_{t}^{x}\right) D_{V_{2}}\left(\nabla_{u_{1}} X_{t}^{x}\right)\right] .
\end{aligned}
$$

Combining the above relations, we immediately obtain the first relation in the proposition. The second relation can immediately be obtained from the first one.

Correction to the proofs of (7.1) and (7.2) in [1]: The conclusions of (7.1) and (7.2) still hold under the strengthened Assumption 2.1., but we need to estimate the extra terms related to $\mathcal{R}_{u_{2}, u_{1}}^{x}(t)$. From the second relation in the above proposition, we have

$$
\begin{aligned}
\left|\nabla_{u_{2}} \nabla_{u_{1}} f(x)\right| \leq & \int_{0}^{\infty} e^{-t}\left|\mathbb{E}\left\{\left[\nabla_{u_{1}} f\left(X_{t}^{x}\right)-\nabla_{u_{1}} h\left(X_{t}^{x}\right)\right] \mathcal{I}_{u_{2}}^{x}(t)\right\}\right| \mathrm{d} t \\
& +\int_{0}^{\infty} e^{-t}\left|\mathbb{E}\left\{\left[\nabla f\left(X_{t}^{x}\right)-\nabla h\left(X_{t}^{x}\right)\right] \mathcal{R}_{u_{1}, u_{2}}^{x}(t)\right\}\right| \mathrm{d} t .
\end{aligned}
$$

Since we have shown in the original proof that

$$
\int_{0}^{\infty} e^{-t}\left|\mathbb{E}\left\{\left[\nabla_{u_{1}} f\left(X_{t}^{x}\right)-\nabla_{u_{1}} h\left(X_{t}^{x}\right)\right] \mathcal{I}_{u_{2}}^{x}(t)\right\}\right| \mathrm{d} t \leq C_{\theta}\|\nabla h\|\left|u_{1}\right|\left|u_{2}\right|,
$$

it remains to bound the second integral. By [1, (5.7), (5.17)], we immediately obtain

$$
\int_{0}^{\infty} e^{-t}\left|\mathbb{E}\left\{\left[\nabla f\left(X_{t}^{x}\right)-\nabla h\left(X_{t}^{x}\right)\right] \mathcal{R}_{u_{1}, u_{2}}^{x}(t)\right\}\right| \mathrm{d} t \leq C_{\theta}\|\nabla h\|\left|u_{1}\right|\left|u_{2}\right| .
$$


Combining the previous three inequalities, we conclude that $[1,(7.1)]$ still holds true.

To prove $[1,(7.2)]$, we have

$$
\nabla_{u_{2}} \nabla_{u_{1}} f(x+\varepsilon u)-\nabla_{u_{2}} \nabla_{u_{1}} f(x)=\int_{0}^{\infty} e^{-t} \Psi \mathrm{d} t+\int_{0}^{\infty} e^{-t} \Phi \mathrm{d} t=J_{1}+J_{2}
$$

where

$$
\begin{aligned}
\Psi= & \mathbb{E}\left\{\left[\nabla_{u_{1}} f\left(X_{t}^{x+\varepsilon u}\right)-\nabla_{u_{1}} h\left(X_{t}^{x+\varepsilon u}\right)\right] \mathcal{I}_{u_{2}}^{x+\varepsilon u}(t)\right\} \\
& -\mathbb{E}\left\{\left[\nabla_{u_{1}} f\left(X_{t}^{x}\right)-\nabla_{u_{1}} h\left(X_{t}^{x}\right)\right] \mathcal{I}_{u_{2}}^{x}(t)\right\} . \\
\Phi= & \mathbb{E}\left\{\left[\nabla f\left(X_{t}^{x+\varepsilon u}\right)-\nabla h\left(X_{t}^{x+\varepsilon u}\right)\right] \mathcal{R}_{u_{1}, u_{2}}^{x+\varepsilon u}(t)\right\} \\
& -\mathbb{E}\left\{\left[\nabla f\left(X_{t}^{x}\right)-\nabla h\left(X_{t}^{x}\right)\right] \mathcal{R}_{u_{1}, u_{2}}^{x}(t)\right\} .
\end{aligned}
$$

We have shown in the original proof that

$$
\left|J_{1}\right| \leq C_{\theta}|| \nabla h|||\varepsilon|(|\log | \varepsilon|| \vee 1)\left|u_{1}\right|\left|u_{2}\right|
$$

We prove below that

$$
\left|J_{2}\right| \leq C_{\theta}|| \nabla h\left|\|\varepsilon\| u_{1}\right|\left|u_{2}\right||u|
$$

Combining the estimates of $J_{1}$ and $J_{2}$, we immediately get that $[1,(7.2)]$ still holds true.

Let us show the above bound about $J_{2}$. Write

$$
J_{2}=J_{2,1}+J_{2,2},
$$

with

$$
\begin{aligned}
& J_{2,1}=\int_{0}^{\infty} e^{-t} \mathbb{E}\left\{\left[\nabla f\left(X_{t}^{x+\varepsilon u}\right)-\nabla h\left(X_{t}^{x+\varepsilon u}\right)\right]\left[\mathcal{R}_{u_{1}, u_{2}}^{x+\varepsilon u}(t)-\mathcal{R}_{u_{1}, u_{2}}^{x}(t)\right]\right\} \mathrm{d} t, \\
& J_{2,2}=\int_{0}^{\infty} e^{-t} \mathbb{E}\left\{\left[\nabla f\left(X_{t}^{x+\varepsilon u}\right)-\nabla h\left(X_{t}^{x+\varepsilon u}\right)-\nabla f\left(X_{t}^{x}\right)+\nabla h\left(X_{t}^{x}\right)\right] \mathcal{R}_{u_{1}, u_{2}}^{x}(t)\right\} \mathrm{d} t,
\end{aligned}
$$

For $J_{2,1}$, observe

$$
J_{2,1}=\varepsilon \int_{0}^{\infty} e^{-t} \int_{0}^{1} \mathbb{E}\left\{\left[\nabla f\left(X_{t}^{x+\varepsilon u}\right)-\nabla h\left(X_{t}^{x+\varepsilon u}\right)\right] \nabla_{u} \mathcal{R}_{u_{1}, u_{2}}^{x+s \varepsilon u}(t)\right\} \mathrm{d} s \mathrm{~d} t,
$$

which, together with Lemma 0.1, immediately gives

$$
\left|J_{2,1}\right| \leq C_{\theta}|\varepsilon|(\|\nabla f\|+\|\nabla h\|) \leq C_{\theta}|\varepsilon|\|\nabla h\|\left|u \| u_{1}\right|\left|u_{2}\right| .
$$


For $J_{2,2}$, we have

$$
\begin{aligned}
J_{2,2} & =\varepsilon \int_{0}^{\infty} e^{-t} \int_{0}^{1} \mathbb{E}\left\{\nabla\left[\nabla f\left(X_{t}^{x+s \varepsilon u}\right)-\nabla h\left(X_{t}^{x+s \varepsilon u}\right)\right] \nabla_{u} X_{t}^{x+s \varepsilon u} \mathcal{R}_{u_{1}, u_{2}}^{x}(t)\right\} \mathrm{d} s \mathrm{~d} t \\
& =\varepsilon \int_{0}^{\infty} e^{-t} \int_{0}^{1} \mathbb{E}\left\{\nabla\left[\nabla f\left(X_{t}^{x+s \varepsilon u}\right)-\nabla h\left(X_{t}^{x+s \varepsilon u}\right)\right] D_{V} X_{t}^{x+s \varepsilon u} \mathcal{R}_{u_{1}, u_{2}}^{x}(t)\right\} \mathrm{d} s \mathrm{~d} t \\
& =\varepsilon \int_{0}^{\infty} e^{-t} \int_{0}^{1} \mathbb{E}\left\{D_{V}\left[\nabla f\left(X_{t}^{x+s \varepsilon u}\right)-\nabla h\left(X_{t}^{x+s \varepsilon u}\right)\right] \mathcal{R}_{u_{1}, u_{2}}^{x}(t)\right\} \mathrm{d} s \mathrm{~d} t \\
& =\varepsilon\left(J_{2,2,1}-J_{2,2,2}\right)
\end{aligned}
$$

where the last equality is by $[1,(5.14),(5.9),(5.11)]$ and

$$
\begin{aligned}
& J_{2,2,1}=\int_{0}^{\infty} e^{-t} \int_{0}^{1} \mathbb{E}\left\{\left[\nabla f\left(X_{t}^{x+s \varepsilon u}\right)-\nabla h\left(X_{t}^{x+s \varepsilon u}\right)\right] \mathcal{R}_{u_{1}, u_{2}}^{x}(t) \mathcal{I}_{u}^{x+s \varepsilon u}(t)\right\} \mathrm{d} s \mathrm{~d} t \\
& J_{2,2,2}=\int_{0}^{\infty} e^{-t} \int_{0}^{1} \mathbb{E}\left\{\left[\nabla f\left(X_{t}^{x+s \varepsilon u}\right)-\nabla h\left(X_{t}^{x+s \varepsilon u}\right)\right] D_{V} \mathcal{R}_{u_{1}, u_{2}}^{x}(t)\right\} \mathrm{d} s \mathrm{~d} t .
\end{aligned}
$$

By Lemma 0.1 and $[1,(5.18)]$,

$$
\begin{aligned}
\left|J_{2,2,2}\right| & \leq C_{\theta}(\|\nabla f\|+\|\nabla h\|)\left|u_{1}\right|\left|u_{2}\right||u| \leq C_{\theta}\|\nabla h\|\left|u_{1}\right|\left|u_{2}\right||u| \\
\left|J_{2,2,1}\right| & \leq C_{\theta}(\|\nabla f\|+\|\nabla h\|)\left|u_{1}\right|\left|u_{2}\right| \int_{0}^{\infty} e^{-t} \int_{0}^{1} \mathbb{E}\left[\left|\mathcal{I}_{u}^{x+s \varepsilon u}(t)\right|\right] \mathrm{d} s \mathrm{~d} t \\
& \leq C_{\theta}\|\nabla h\|\left|u_{1}\left\|u_{2}\right\| u\right| .
\end{aligned}
$$

Combining the estimates above, we immediately obtain the bound of $J_{2}$.

Due to the new assumption on $g$, [1, Remark 3.2] should be revised as

Remark 3.2 Gorham et. al. (see [18] in [1]) recently put forward a method to measure sample quality with diffusions by a Stein discrepancy, in which the same Stein equation as (3.1) has to be considered. Under the assumption that $g$ is third-order differentiable, they used the Bismut-Elworthy-Li formula (see [16] in [1]), together with smooth convolution and interpolation techniques, to prove a bound on the first, second, and $(3-\epsilon)$ th derivative of $f$ for $\epsilon>0$. They can also obtain the bound (3.4) by their approach (personal communication (see [24] in [1]) after their reading our paper on ArXiv) together with a limiting argument.

Acknowledgements We thank Jim Dai and James Thompson for pointing out the errors.

\section{Reference}

1. Fang, X., Shao, Q.M., Xu, L.: Multivariate approximations in Wasserstein distance by Stein's method and Bismut's formula. Probab. Theory Relat. Fields (2019) (to appear)

Publisher's Note Springer Nature remains neutral with regard to jurisdictional claims in published maps and institutional affiliations. 